\title{
Factores de riesgos psicosociales extralaborales en personal administrativo universitario ${ }^{1}$
}

Institución: Universidad de la Costa, Colombia

\author{
Carmen Helena Romero-Díaz ${ }^{2}$ \\ Rosana Beleño Navarro ${ }^{3}$ \\ María Ucros Campo ${ }^{4}$ \\ Ana Echeverría González ${ }^{5}$ \\ Silvana Lasprilla Fawcett ${ }^{6}$
}

\section{COMO CITAR}

Romero-Díaz, C.H., Beleño, R., Ucros, M., Echeverría, A. y Lasprilla, S.. (2016). Factores de riesgos psicosociales extralaborales en personal administrativo universitario. Rev. Enfermería Actual en Costa Rica, 31, 1-15. DOI: http://dx.doi.org/10.15517/revenf.v0i31.22083

\section{RESUMEN}

Introducción. La presente investigación tuvo como objetivo caracterizar los factores de riesgo psicosociales extralaborales del personal administrativo de la Corporación Universidad de la Costa - CUC y la determinación de su nivel de estrés.

Método. A partir de un censo se determinó la población, formada por 227 trabajadores. Es una investigación de campo, descriptiva, transaccional, de diseño no experimental. En la recolección de información se aplicó los instrumentos para la evaluación de factores de riesgos psicosociales, emitida reglamentariamente por el Ministerio de Protección Social de Colombia.

Resultados. Se evidenciaron los principales factores de este tipo de riesgo, así como su nivel de estrés, presentes en los trabajadores administrativos de la institución, que demuestran que 68 trabajadores sumaron los niveles de estrés en las categorías muy alto y alto, 76 sujetos agruparon a los niveles medio y bajo, mientras que los 83 restantes, presentaron riesgo muy bajo.

Conclusión. En primer lugar, prevalece el factor de riesgo "desplazamiento vivienda-trabajo-vivienda", en segundo la "situación económica del grupo familiar" y, en tercer lugar, las "características de la vivienda y su entorno". Existe una considerable alarma para el sostenimiento de la buena salud laboral del personal administrativo de la universidad, cuando los estamentos nacionales estiman que a partir de la categoría de nivel de estrés bajo, se deben acometer intervenciones especiales y cuando se elevan a partir del nivel medio se comprometen valores epidemiológicos de la salud pública de la nación.

Palabras clave: estrés, factores-psicosociales, personal-administrativo.

\footnotetext{
${ }^{1}$ Fecha de recepción: 27 de noviembre del 2015

Fecha de aceptación: 15 febrero del 2016

${ }^{2}$ Docente. Investigadora. Facultad de Humanidades. Universidad de la Costa Barranquilla, Colombia. Correo electrónico: cromero11@cuc.edu.co

${ }^{3}$ Docente investigador. Universidad de la Costa. Barranquilla, Colombia. Correo electrónico: rbeleno2@cuc.edu.co

${ }^{4}$ Docente investigador. Universidad de la Costa. Barranquilla, Colombia. Correo electrónico: mucros3@cuc.edu.co

${ }^{5}$ Docente investigador. Universidad de la Costa. Barranquilla, Colombia. Correo electrónico: aecheverria@cuc.edu.co

${ }^{6}$ Docente investigador. Universidad de la Costa. Barranquilla, Colombia. Correo electrónico: $\underline{\text { slaspril1 @ cuc.edu.co }}$
} 


\title{
Psychosocial risk factors outside in university administrative personnel $^{1}$
}

\author{
Institution: De la Costa University, Colombia
}

\author{
Carmen Helena Romero-Díaz ${ }^{2}$ \\ Rosana Beleño Navarro ${ }^{3}$ \\ María Ucros Campo ${ }^{4}$ \\ Ana Echeverría González ${ }^{5}$ \\ Silvana Lasprilla Fawcett ${ }^{6}$
}

\section{CITED}

Romero-Díaz, C.H., Beleño, R., Ucros, M., Echeverría, A. y Lasprilla, S.. (2016). Psychosocial risk by outside working factors of administrative staff university. Rev. Enfermería Actual en Costa Rica, 31, 1-15.http://dx.doi.org/10.15517/revenf.v0i31.22083

\begin{abstract}
Introduction. This study aimed to characterize the psychosocial risk by outside working factors of administrative staff of the University Corporation of the Coast - CUC and the determination of their level of stress.

Method. From a census was determined the population, which was formed by 227 workers. It is a research, descriptive, transactional with non-experimental design. The collection of information used instruments for the evaluation of factors of psychosocial risks, that it is regulations of the Ministry of Social protection of Colombia.

Results. It showed the main factors of this type of risk, as well as your stress level, present in the administrative employees of the institution, showing that 68 workers joined in the categories high and high stress levels, 76 subjects were grouped by levels the medium and low, while the remaining 83 , had very low risk.

Conclusion. Firstly, prevailed the risk factor about "transportation of home - work - home", the second factor "Economic situation of the family group", and thirdly the "characteristics of the dwelling and its surroundings". There is a considerable alarm for the maintenance of the good health of the administrative staff of the University, when the levels stress are in the category of stress low and it need should undertake special interventions and with the rising from the middle level undertake epidemiological values of the nation's public health.
\end{abstract}

Key words: administrative-staff, psychosocial-factors, stress.

\footnotetext{
${ }^{1}$ Date of receipt: November 27, 2015

Date of acceptance: February 15, 2016

${ }^{2}$ Professor. Researcher. Faculty of Humanities. De la Costa University. Barranquilla, Colombia. E-mail: cromero11@ @ cuc.edu.co

${ }^{3}$ Professor. Researcher. De la Costa University. Barranquilla, Colombia. E-mail: rbeleno2@cuc.edu.co

${ }^{4}$ Professor. Researcher. De la Costa University. Barranquilla, Colombia. E-mail: mucros3@cuc.edu.co

${ }^{5}$ Professor. Researcher. De la Costa University. Barranquilla, Colombia. E-mail: $\underline{\text { aecheverria@cuc.edu.co }}$

${ }^{6}$ Professor. Researcher. De la Costa University. Barranquilla, Colombia. E-mail: slaspril1@cuc.edu.co
} 


\title{
Intralaborales psicossociais fatores de risco em universidade pessoal administrativo ${ }^{1}$
}

Instituição: Universidade da la Costa, Colombia

\author{
Carmen Helena Romero-Díaz ${ }^{2}$ \\ Rosana Beleño Navarro ${ }^{3}$ \\ María Ucros Campo ${ }^{4}$ \\ Ana Echeverría González ${ }^{5}$ \\ Silvana Lasprilla Fawcett ${ }^{6}$
}

\section{CITAÇÃO}

Romero-Díaz, C.H., Beleño, R., Ucros, M., Echeverría, A. y Lasprilla, S.. (2016 Intralaborales psicossociais fatores de risco em universidade pessoal administrativo. Rev. Enfermería Actual en Costa Rica, 31, 1-15. http://dx.doi.org/10.15517/revenf.v0i31.22083

\section{RESUMO}

Introdução. A presente investigação teve como objetivo caracterizar os fatores de risco psicosociais externos ao trabalho do pessoal administrativo da Corporação Universidade da Costa - CUC e a determinação de seu nível de estresse.

Método. A partir de um censo se determinou a população, formada por 227 trabalhadores. É uma investigação de campo, descritiva, transacional, de desenho não experimental. Na recoleta de informação se aplicaram os instrumentos para a avaliação de fatores de riscos psicosociais, emitida regulamentariamente pelo Ministério de Proteção Social da Colômbia.

Resultado. Foram evidenciados os principais fatores deste tipo de risco, assim como o nível de estresse presentes nos trabalhadores administrativos da instituição, que demonstram que 68 trabalhadores somaram os níveis de estresse nas categorias muito alto e alto, 76 sujeitos agruparam aos níveis médio e baixo, enquanto que os 83 restantes, apresentaram risco muito baixo.

Conclusão. Em primeiro lugar, prevalece o fator de risco "deslocamento moradia-trabalho-moradia", em segundo a "situação econômica do grupo familiar" e, em terceiro lugar, as "características da moradia e seu entorno". Existe um considerável alarmante para sustentar a boa saúde no trabalho do pessoal administrativo da universidade, quando os estamentos nacionais estimam que a partir da categoria de nível de estresse baixo, se devem realizar intervenções especiais e quando se elevam a partir do nível médio se comprometem valores epidemiológicos da saúde pública da nação.

Palavras chave: estresse, fatores-psicosociais, pessoal-administrativo.

\footnotetext{
${ }^{1}$ Data de recebimento: 27 de novembro de 2015

Data de aceitação: 15 de fevereiro de 2016

${ }^{2}$ Professor. Investigador. Faculdade de Ciências Humanas. Universidade de la Costa. Barranquilla, Colombia. Correio eletrônico: cromero11@cuc.edu.co

${ }^{3}$ Professor. Investigador. Universidade da la Costa. Barranquilla, Colombia. Correio eletrônico: rbeleno2@cuc.edu.co

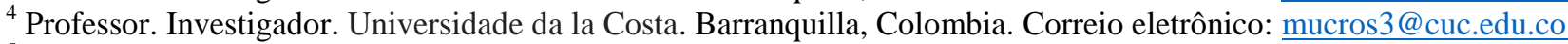

${ }^{5}$ Professor. Investigador. Universidade da la Costa. Barranquilla, Colombia. Correio eletrônico: aecheverria@cuc.edu.co

${ }^{6}$ Professor. Investigador. Universidade da la Costa. Barranquilla, Colombia. Correio eletrônico: slaspril1@ @uc.edu.co
} 


\section{Revista Electrónica Enfermeria Actual en costa Rica}

\section{INTRODUCCIÓN}

Los factores de riesgo psicosociales han sido definidos por la Organización Internacional del Trabajo (OIT, 1986) como las interacciones entre el trabajo, su medio ambiente, así como la satisfacción en el trabajo y las condiciones de organización, teniendo en cuenta las capacidades del trabajador, sus necesidades, su cultura y su situación personal fuera del trabajo que pueden influir en el estado de salud y bienestar de las personas, hasta determinar su nivel de rendimiento, además de su satisfacción en el trabajo.

Tal dimensión comprende los aspectos psicológicos y sociales que subyacen en la actividad laboral que desempeña un trabajador y afectan la calidad de vida de los mismos. Es por ello que se incluyen aspectos afectivos, competencias desarrolladas en la actividad laboral y las expectativas que esto demanda (MorenoJiménez y Garrosa-Hernández, 2009). Estos factores psicosociales pueden favorecer o perjudicar la actividad laboral y la calidad de vida laboral de las personas. En el primer caso, fomentan el desarrollo personal de los individuos, mientras que cuando son desfavorables perjudican su salud y su bienestar (Gil-Monte, 2012). En este caso hablamos de riesgo psicosocial, que es fuente de estrés laboral, o estresor y que tiene el potencial de causar daño psicológico, físico, o social a los individuos. (Gil-Monte, 2009)

De acuerdo con la definición otorgada por el Ministerio de Protección Social en Colombia (MPS), en su Resolución 2646 de 2008, los factores psicosociales intralaborales o condiciones intralaborales son entendidas como aquellas características del trabajo y de su organización que influyen en la salud y el bienestar del individuo. En este sentido, se abordará cuatro dominios de factores psicosociales intralaborales: demandas de trabajo, control sobre el trabajo, liderazgo y relaciones sociales en el trabajo, y recompensa.

Desde lo connotado como aspectos extralaborales, el trabajador no puede verse como un ser "fragmentado" ya que sobre él influyen diferentes condiciones determinadas por su historia personal y laboral, las actividades desarrolladas en el tiempo libre (extralaboral) y su interacción social especialmente en el ámbito familiar (Guerrero, 2003). Así mismo, el Instituto Nacional de Seguridad e Higiene en el Trabajo (2001), señala que las condiciones de trabajo no pueden ser analizadas si no se incluyen las condiciones de la vida extralaboral, lo cual es confirmado por el Ministerio de la Protección Social de Colombia (2010), entidad que afirma que las condiciones extralaborales "comprenden los aspectos del entorno familiar, social y económico del trabajador. A su vez, abarcan las condiciones del lugar de vivienda, que pueden influir en la salud y bienestar del individuo" (p.26).

En cuanto a los estratos sociales, en Colombia se reconoce en una escala que va desde el nivel 1 hasta el nivel 6, en los que el más bajo se refiere a una condición de vida de extrema necesidad, asociada a una población en estado de vulnerabilidad. El estrato 2 representa a personas de escasos recursos económicos y a partir del estrato 3 se consideran a las personas con mejor estado de vida y mejor posibilidad de trabajo. Cuando el estrato sube desde el nivel 4, 5 y 6, se refiere a personas que van desarrollando una condición de vida de mejor calidad y solvencia económica.

Debido a las consecuencias de los factores psicosociales en quienes trabajan, se produce un desgaste en el proceso salud-enfermedad de esta población, por el esfuerzo cotidiano de la jornada laboral que les produce gran cantidad de estrés sobre lo que cabe mencionar que un aumento desmesurado del estrés disminuye la eficacia y 


\section{Revista Electrónica Enfermeria Actual en costa Rica}

aumenta el riesgo en la salud (Sánchez, 2010). En torno al tema, hay que destacar que el estrés se ha reconocido como uno de los factores de riesgo psicosocial, debido a formas de trabajo centradas dominantemente en el individuo y en la relación con su entorno (Fernández, 2012). El término estrés ha sido definido por Selyle en 1956 ante la Organización Mundial de la Salud (OMS), como la respuesta no especificada del organismo a cualquier demanda del exterior (citado por Hernández, Soria y Silla, 2003). Para Sotillo, (2000), se conceptualiza como el conjunto de fenómenos que suceden en el organismo y salud del trabajador, con la participación de los agentes estresantes lesivos derivados directamente del trabajo. Así mismo, Richard Lazarus, en su teoría lo define como un proceso sostenido en el tiempo, en el que de manera regular el individuo percibe desbalance entre las exigencias de una situación y los recursos con los que cuenta para hacer frente a dicha situación (Lazarus, 1999).

La Universidad Pontificia Javeriana en el Subcentro de Seguridad Social y Riesgos Profesionales (citado por Díaz, 2011), lo define como un conjunto de reacciones de carácter psicológico, emocional y comportamental, que se produce cuando el individuo debe de enfrentar demandas derivadas de su interacción con el medio (factores de riesgo psicosocial), ante las cuales su capacidad de afrontamiento es insuficiente, causando un desbalance que afecta el bienestar e incluso su salud.

Para Marulanda (2007), el estrés laboral ha generado impacto en la salud de los trabajadores, en su productividad y en el ambiente de trabajo, al punto de conceptualizarlo como el desbalance percibido por el trabajador entre las condiciones psicosociales presentes en el contexto del trabajo y sus capacidades, características y expectativas individuales. Por su parte, Mamani, Obando, Uribe y Vivanco (2007), lo relacionan con el contenido de trabajo, el grado de responsabilidad, el conflicto y la ambigüedad de rol, los contactos sociales y el clima de la organización, la carga de trabajo, la necesidad de mantenimiento y desarrollo de una cualificación profesional, los horarios irregulares, la violencia hacia el profesional, el ambiente físico en el que se realiza el trabajo, no tener oportunidad de exponer la quejas e inseguridad en el empleo.

Fernández (2012), expone que los principales estresores son característicos del entorno laboral y de las características del trabajo, cuyas consecuencias pueden ser a nivel personal y a nivel organizacional. Manifiesta que a nivel personal las consecuencias serían fisiológicas y psíquicas y sobre la organización ocasionaría absentismo laboral, falta de implicación y rendimiento escaso. Así mismo, Villegas y Santamaría (1999) agregan que los sujetos casados suelen tener un estilo de vida más estable y una perspectiva de vida diferente a los solteros. Los sujetos solteros podrían tender a usar el trabajo como fuente de vida social y esto le llevaría a implicarse excesivamente con la gente del ámbito laboral y, por tanto, a un mayor riesgo de sufrir estrés y el síndrome de quemarse en el trabajo.

Por su parte, Arias et al. (2008) estudian el síndrome de desgaste profesional o de burnout en el personal de enfermería, lo cual también se reconoce como consecuencia de elevados niveles de estrés. En su investigación, se logró determinar la prevalencia de este síndrome y de sus síntomas, como el cansancio emocional, la despersonalización y la falta de realización personal, entre los profesionales de enfermería. Sus resultados revelaron que las relaciones interpersonales, el ambiente físico, la situación laboral y el bienestar del cliente han provocado el desgaste profesional y un consecuente déficit en las tareas profesionales, debido al estrés que han sufrido. 


\section{Revista Electrónica Enfermeria Actual en costa Rica}

Sánchez (2009) estudió los factores psicosociales de los trabajadores de una empresa del sector público, con el objetivo de comparar los factores intralaborales y extralaborales. La metodología estuvo basada en un estudio cualitativo utilizando el método estructural investigación acción, con una población constituida por una muestra de 12 hombres y 12 mujeres. En este estudio, Sánchez (2009) empleó el Índice de Cornell para conocer si la población presentaba síntomas relacionados con el estrés. Los resultados evidenciaron que los empleados presentaron riesgos intralaborales y, en cuanto a los factores de riesgo extralaborales, las preocupaciones familiares de cuido y atención de la familia representan un obstáculo para ejecutar con satisfacción su trabajo.

En relación con los diversos factores y niveles de estrés, Londoño et al. (2010) agregan que los problemas de vivienda representan un factor de riesgo para la depresión: en países como Colombia, este tipo de problema también está relacionado con la dificultad de enfrentar el diario vivir con los créditos hipotecarios y demás gastos ligados a la vivienda. A esto se unen las dificultades para el desplazamiento vivienda-trabajo-vivienda, que también se convierte en el factor de riesgo que incrementa el estrés del trabajador; al respecto, Gutiérrez y García (2005) expone que las metrópolis actuales se caracterizan por una movilidad creciente con mayor número de viajes por persona, incrementando las distancias recorridas en los viajes y el aumento constante en el uso de transporte privado. Según los expertos la ciudad de Barranquilla creció vertiginosamente quedándose atrás en obras de infraestructura vial y la Empresa de Transporte y Tránsito Metropolitano de Barranquilla (Metrotránsito S.A.) acepta que la situación de la movilidad en la ciudad es caótica, debido a la falta de planeación urbana (Cámara Colombiana de la Infraestructura, 2005).

Por su parte, en cuanto al componente económico de las personas que trabajan, Gómez y Rodríguez (1997) encontraron que un factor importante para la aparición de depresión estaba relacionado con los ingresos familiares. En este aspecto se describió una relación inversa entre ingresos y depresión: cuando todas las situaciones económicas eran satisfechas, el porcentaje de personas que estaba deprimida disminuía y aumentaba en la medida en que los ingresos familiares se iban haciendo menores.

Orozco (2010) estudia los factores psicosociales del ambiente laboral que caracterizan a funcionarios con contratación a término fijo e indefinido de una universidad privada de Cali - Colombia. Fue un estudio de tipo descriptivo transversal que aplicó un instrumento cuantitativo denominado ISTAS $21^{1}$, con el cual se evalúa los riesgos psicosociales en el trabajo para empresas de 25 y más trabajadores. Esta investigación permitió identificar los factores de riesgo, así como otros factores protectores, entendidos como el sentido del trabajo, integración en la empresa, posibilidades de desarrollo, claridad de rol, calidad de liderazgo, apoyo social y refuerzo. Este estudio exhorta a tener en cuenta el manejo de las emociones, capacidad de autocontrol, adecuada atención al cliente, buenas relaciones sociales, entre otros aspectos, ya que sin ello no se podría garantizar la calidad del servicio a la comunidad educativa.

También, cabe mencionar el trabajo de Cano (2012), quien realizó un estudio basado en la identificación, medición y evaluación de los factores de riesgo psicosocial, en el personal de trabajadores de la Universidad San Francisco, utilizando el método FPSICO, aplicado a docentes, administrativos y los operativos. Los resultados mostraron que el personal administrativo y docente mantiene la tendencia de exposición a riesgos psicosociales

\footnotetext{
${ }^{1}$ Instrumento para la prevención de riesgos psicosociales
} 


\section{Revista Electrónica Enfermeria Actual en costa Rica}

elevados, mientras que en el grupo operativo predomina los psicosociales moderados. Esto permitió tomar medidas de intervención para mejorar las condiciones laborales con acciones preventivas.

Por su parte, Aguirre y Guevara (2012) desarrollaron una investigación para determinar los factores que afectan el desempeño laboral de oficiales de Tránsito y Seguridad. La población estuvo formada por 57 oficiales de tránsito y seguridad, a quienes se les aplicó los instrumentos basados en una guía de observación y un cuestionario. Como resultado se evidenció una alta incidencia de enfermedades crónicas, que empeoran al exponerse a factores propios del trabajo, así como por la práctica de inadecuados estilos de vida como el tabaquismo, una mala alimentación y el sedentarismo. Además, prevalecieron factores de riesgo como el grupo etario, jornadas laborales excesivas, horarios rotativos, así como alta movilidad por el campus. Como principal hallazgo, se encontró que las causas más frecuentes de incapacidad laboral son las caídas y la gripe. Se concluye que los beneficios de un ambiente de trabajo saludable no se reducen al fortalecimiento de la salud del trabajador (a), sino a brindar un aporte positivo a la productividad e incrementar la motivación laboral, el espíritu de trabajo, la satisfacción en el trabajo y la calidad de vida general.

Desde una mirada de los constructos legales, la Organización Internacional del Trabajo (OTI), considera relevante emprender el desarrollo de políticas para la creación de condiciones favorables de trabajo, a partir de su declaración relativa a los principios y derechos fundamentales en el trabajo. Colombia se suma a este enunciado, a través de la publicación del convenio sobre el marco promocional para la seguridad y salud en el trabajo, desde el 20 febrero de 2009 (OIT, 2006), en el que se expone aspectos importantes, como la Política Nacional sobre Seguridad y Salud en el Trabajo y el Medio Ambiente de Trabajo y el Sistema Nacional de Seguridad y Salud en cuanto a una infraestructura para gestión de tales políticas, entre otros aspectos.

En este sentido, las instituciones de educación superior en Colombia, apuestan al cumplimiento de las normas respectivas, a partir de la generación de políticas internas que les permitan cumplir con las disposiciones de la Constitución Política y del Ministerio de la Protección Social. En tanto, el Ministerio de Educación Nacional en 1992 a través de la Ley 30 de 1992 (MEN, 1992) organiza el servicio público de la educación superior, exhortando a establecer los departamentos de bienestar universitario, con políticas que encaminen el desarrollo armónico del personal estudiantil, docente y administrativo, desde lo físico, psicoafectivo, espiritual y social en general.

Las observaciones permanentes sobre el ambiente laboral de los trabadores administrativos en la Corporación Universidad de la Costa, ha mostrado la recurrencia de visitas al Departamento de Bienestar Universitario, en donde se presta, entre otros aspectos, consultas con ayudas orientativas psicológicas, por medio de las cuales se ha verificado la suma progresiva de trabajadores con manifestaciones de estrés de gran significancia y consecuentes patologías médicas relacionadas que varían de una persona a otra. Este contexto situacional, con personas del trabajo administrativo de la institución que manifiestan alteraciones de su estado de salud físico y psicológico, representa el problema objeto de estudio, por lo cual la universidad solicitó investigar los posibles factores estresores que, desde el punto de vista extralaboral, pudieran desencadenar niveles elevados de estrés en sus trabajadores.

Dado lo anterior, el objetivo de esta investigación fue caracterizar los factores de riesgo psicosociales extralaborales del personal administrativo de la Corporación Universidad de la Costa y la determinación de su 


\section{Revista Electrónica Enfermeria Actual en costa Rica}

nivel de estrés, con lo cual se aspira tener las bases fundamentales que permitan encaminar las intervenciones oportunas que sean requeridas por el personal administrativo, de acuerdo con sus necesidades latentes.

\section{MATERIALES Y MÉTODOS}

Para el momento del estudio, la población estuvo constituida por el personal administrativo activo de la Corporación Universidad de la Costa - CUC, con 227 personas que se consideraron como censo, las cuales participaron de manera voluntaria. Teniendo en consideración el conjunto de instrumentos para evaluar los factores de riesgo psicosocial en las instituciones, que la nación colombiana establece reglamentariamente a través del Ministerio de la Protección Social, se procedió a aplicarlo al personal administrativo de la universidad, descrito anteriormente. Enmarcados en el positivismo, con una investigación de corte cuantitativo, se desarrolló un trabajo de campo, transaccional, de diseño no experimental.

Los factores de riesgo psicosocial extralaborales, en este sentido, están clasificados por medio de siete dimensiones que son: características de la vivienda y de su entorno, comunicación y relaciones interpersonales, desplazamiento vivienda-trabajo-vivienda, influencia del entorno extralaboral sobre el trabajo, relaciones familiares, situación económica del grupo familiar y tiempo fuera del trabajo. Cada una de estas dimensiones son evaluadas y sus resultados se trabajan automáticamente con estadística descriptiva, encaminados por las directrices gubernamentales y sus disposiciones reglamentarias.

En cuanto al nivel de estrés, el mismo grupo de instrumentos expedido por la nación, dispone de cuatro agrupaciones globales de estrés: fisiológico, social, intelectual y del trabajo, además del psicoemocional. Se utilizó el grupo de instrumentos para evaluar los factores de riesgo psicosocial diseñados y validados por el Ministerio de la Protección Social de Colombia, a través de la Pontificia Universidad Javeriana, Subcentro de Seguridad Social y Riesgos Profesionales (MPS, 2010). En esta investigación, se consideró los factores de riesgo psicosocial extralaborales, en virtud de que los de tipo intralaboral forman parte de otro momento investigativo y la necesidad emergente de la institución hizo el llamado al desarrollo de este trabajo.

El cuestionario utilizado en esta investigación, está compuesto por los factores psicosociales extralaborales o externos a la organización y las condiciones individuales o características intrínsecas al trabajador, los cuales, en una interrelación dinámica, mediante percepciones y experiencias, influyen en la salud y el desempeño de las personas. La confiabilidad del cuestionario se estableció a través del cálculo de coeficientes de Alfa de Cronbach que alcanzaron un nivel de 0,937. El cuestionario para la evaluación del estrés fue diseñado por Castillo y Villalobos (1996), con el respaldo del Ministerio de Trabajo y Seguridad Social, validado para población colombiana en el año de 1996. Este cuestionario está diseñado con escala Likert, por tanto, plantea una serie de afirmaciones para que el individuo reporte la frecuencia con que ocurren estas situaciones en su vida cotidiana. Las opciones de respuesta son: siempre, casi siempre, a veces o nunca. Cada una de estas opciones de respuesta adquiere un valor diferente, dependiendo de la pregunta y del área de ajuste a evaluar (MPS, 2010).

Los baremos ${ }^{1}$ de calificación de los cuestionarios mencionados permiten clasificar los niveles de riesgo en muy alto, alto, medio, bajo y muy bajo. En el caso del Cuestionario de Estrés, se estima tres categorías: estrés 


\section{Revista Electrónica Enfermeria Actual en costa Rica}

alto, estrés medio y estrés bajo. Cada uno de los posibles niveles de riesgo tiene interpretaciones particulares que se reseña a continuación:

- Sin riesgo o riesgo despreciable (muy bajo): ausencia de riesgo o riesgo tan bajo que no amerita desarrollar actividades de intervención.

- Riesgo bajo: no se espera que los factores psicosociales que obtengan puntuaciones de este nivel estén relacionados con síntomas o respuestas de estrés significativas. Las dimensiones y dominios que se encuentren en esta categoría serán objeto de acciones o programas de intervención, a fin de mantenerlos en los niveles de riesgo más bajos posibles.

- Riesgo medio: nivel de riesgo en el que se esperaría una respuesta de estrés moderada. Las dimensiones y dominios que se encuentren en esta categoría ameritan observación y acciones sistemáticas de intervención para prevenir efectos perjudiciales en la salud.

- Riesgo alto: nivel de riesgo que tiene una importante posibilidad de asociación con respuestas de estrés alto, por tanto, las dimensiones y dominios que se encuentren en esta categoría requieren intervención en el marco de un sistema de vigilancia epidemiológica.

- Riesgo muy alto: nivel de riesgo con amplia posibilidad de asociarse a respuestas muy altas de estrés, por consiguiente, las dimensiones y dominios que se encuentren en esta categoría requieren intervención inmediata en el marco de un sistema de vigilancia epidemiológica.

Esta investigación se desarrolló en tres fases fundamentales:

Fase 1: Trámites de autorización y revisión de referentes teórico-científicos: en esta fase, teniendo en cuenta el objeto de estudio, se esclareció la población para el estudio y se procedió a la solicitud formal de los respectivos permisos institucionales para el desarrollo de la investigación. En este sentido, se realizó un acercamiento a las autoridades universitarias y sus funcionarios departamentales para dar a conocer las intenciones del estudio, con el objetivo de que aprobaran su participación voluntaria. Además, se les exhortó sobre la pertinencia y utilidad de la investigación, en beneficio de mantener un clima laboral óptimo que garantice una mejor productividad.

Fase 2: después de haber recibido la aprobación de los administrativos, se aplicó los instrumentos para evaluar los factores de riesgo psicosociales extralaborales a 227 personas. El tiempo estimado para la aplicación del cuestionario osciló entre 30 a 60 minutos por individuo y se realiza, tanto de forma individual, como colectiva, por áreas de trabajo, siempre manteniendo un manejo confidencial de la información.

Fase 3: en esta fase, se procede a calificar el cuestionario e interpretar los datos, los ítems y sus respectivas dimensiones. De esta forma, son calificados con una interpretación en la que a mayor puntaje obtenido, mayor es el riesgo psicosocial, lo cual también aplica para la puntuación total del cuestionario.

Cabe precisar que para efectos de facilitar la calificación del cuestionario, los autores de los instrumentos para la evaluación desarrollaron un aplicativo básico para la captura de los datos, el cual contiene toda la sintaxis necesaria para que sea el sistema de información el que efectúe los siguientes pasos: (1) calificación de los ítems;

\footnotetext{
${ }^{1}$ Tabla numérica que compara el resultado o puntaje directo (P.D.) que obtiene un sujeto en una prueba psicométrica con el grupo al que pertenece.
} 


\section{Revista Electrónica Enfermeria Actual en costa Rica}

(2) obtención de los puntajes brutos; (3) transformación de los puntajes brutos; (4) comparación de los puntajes transformados con las tablas de baremos y obtención del informe individual para cada trabajador.

Cada uno de los ítems del cuestionario debe ser calificado con valores (números enteros) que oscilan entre 0 y 4, los cuales permiten calcular los puntajes brutos de las dimensiones y dominios, así como el puntaje bruto total del cuestionario. Así mismo, cada una de las opciones de respuesta de los ítems (siempre, casi siempre, a veces, casi nunca y nunca) tienen un valor, el cual debe indicar que a mayor puntuación del ítem, mayor riesgo psicosocial. Dichos valores pueden variar de acuerdo con la forma como está planteado cada ítem, así que para un grupo de reactivos una respuesta de siempre tiene un valor igual a cero (0) y una respuesta de nunca tiene un valor de cuatro (4), mientras que otro grupo de reactivos se califica al contrario. Comparados los puntajes transformados con los baremos que les correspondan, según su dimensión, se podrá identificar el nivel de riesgo psicosocial que representan.

Finalmente, luego de realizadas las descripciones y análisis de los resultados, se procedió a la elaboración de las estrategias conducentes a disminuir los factores de riesgo psicosocial extralaboral que permitan favorecer un clima laboral armónico para los trabajadores administrativos de la CUC.

\section{Consideraciones éticas.}

A través de la Resolución 002646 de 2008, del Ministro de la Protección Social (MPS) en Colombia, en concordancia con el artículo 2 del Decreto 614 de 1984 (Presidencia de la República, 1984), el objeto de la salud ocupacional comprende la protección de la persona contra los riesgos relacionados con diferentes agentes, entre los cuales se cita los de tipo psicosocial. En este sentido la Corporación Universidad de la Costa (CUC), se suma desde su ética corporativa a la gestión de investigaciones, para reconocer los posibles factores de riesgos psicosociales de sus trabajadores. En cuanto al desarrollo de esta investigación, las fases del estudio han comprendido los trámites de autorización que ratifican la solicitud de los permisos institucionales inherentes para el desarrollo de la misma considerando, así mismo, el consentimiento de cada uno de los 227 trabajadores, para participar en este estudio.

\section{RESULTADOS}

En la Corporación Universidad de la Costa - CUC, existen factores de riesgo psicosocial extralaborales asociados al estrés de los trabajadores administrativos. Los detalles de los resultados asociados a este hallazgo se presentan a continuación, considerando los datos preliminares de los sujetos, respecto de su condición básica de género, estado civil, estrato social y tipo de vivienda. Los datos evidencian que, de todos los administrativos encuestados, 157 de los sujetos corresponde al género femenino y 70, al masculino. En cuanto al estado civil se observa que 107 sujetos están solteros, 82 casados, 32 en unión libre, 4 sujetos se encuentran separados y los 2 restantes son viudos.

Los resultados del estrato social de los trabajadores administrativos de la CUC revelan que, de los 227 sujetos encuestados, el mayor porcentaje con 79 trabajadores se ubica en el estrato social 3, seguido con 68 en el estrato social 2 y 36 personas en el estrato 1. Finalmente, con menor porcentaje se ubica 32 sujetos en el estrato social 4, seguido de 7 trabajadores en el estrato social 6 y, por último, 5 pertenecientes al estrato social 5. 


\section{Revista Electrónica Enfermeria Actual en costa Rica}

Los datos obtenidos sobre el tipo de vivienda de los trabajadores administrativos de la CUC, manifiestan que 79 personas habitan viviendas de familiares, 74 tienen casa propia y otros 74 , se encuentran en viviendas arrendadas.

Los resultados de los factores psicosociales extralaborales en el personal administrativo de la CUC, presentados en la Tabla 1, develan que en la "dimensión de características de la vivienda y su entorno", 58 se ubican con riesgo muy bajo, 38 de los encuestados en riesgo bajo, 30 están en riesgo medio, 62 presentaron riesgo alto y 39 , muy alto. En la "dimensión de comunicación y relaciones interpersonales", 157 trabajadores se ubican en riesgo muy bajo, 52 presentaron riesgo bajo, 14 obtuvieron riesgo medio y 4 personas presentaron riesgo alto.

Para la "dimensión desplazamiento vivienda-trabajo-vivienda", 58 de los sujetos encuestados presentaron riesgo muy bajo; 32 , riesgo bajo: 37 , riesgo medio; 45 , riesgo alto y 55, riesgo muy alto. En cuanto a la "dimensión influencia del entorno extralaboral sobre el trabajo", 84 trabajadores se encuentran con riesgo muy bajo; 36, con riesgo bajo; 38 en riesgo medio; 40 con riesgo alto y 29, con riesgo muy alto. En la "dimensión de relaciones familiares", 118 de los encuestados presentan riesgo muy bajo; 46, riesgo bajo; 14, riesgo medio; 33, riesgo alto y 16, riesgo muy alto. En la "dimensión situación económica del grupo familiar", 54 trabajadores tuvieron riesgo muy bajo; 34 , riesgo bajo; 58 , riesgo medio; 36 , riesgo alto y 45 , riesgo muy alto. En cuanto a la "Dimensión tiempo fuera del trabajo", 52 trabajadores presentaron riesgo muy bajo; 59, riesgo bajo; 46, riesgo medio; 40, riesgo alto y 30 , riesgo muy alto.

Tabla 1. Corporación Universidad de la Costa. Distribución de factores psicosociales extralaborales en el personal administrativo.

\begin{tabular}{cccccccc}
\hline & $\begin{array}{c}\text { Características } \\
\text { de la vivienda } \\
\text { y su entorno }\end{array}$ & $\begin{array}{c}\text { Comunicación } \\
\text { y relaciones } \\
\text { interpersonales }\end{array}$ & $\begin{array}{c}\text { Desplazamiento } \\
\text { vivienda- } \\
\text { trabajo- } \\
\text { vivienda }\end{array}$ & $\begin{array}{c}\text { Influencia } \\
\text { del entorno } \\
\text { extralaboral } \\
\text { sobre el } \\
\text { trabajo }\end{array}$ & $\begin{array}{c}\text { Relaciones } \\
\text { familiares }\end{array}$ & $\begin{array}{c}\text { Situación } \\
\text { económica } \\
\text { del grupo } \\
\text { familiar }\end{array}$ & $\begin{array}{c}\text { Tiempo } \\
\text { fuera del } \\
\text { trabajo }\end{array}$ \\
\hline $\begin{array}{c}\text { Muy } \\
\text { Alto }\end{array}$ & 39 & 0 & 55 & 29 & 16 & 45 & 30 \\
\hline Alto & 62 & 4 & 45 & 40 & 33 & 36 & 40 \\
\hline Medio & 30 & 14 & 37 & 38 & 14 & 58 & 46 \\
\hline Bajo & 38 & 52 & 32 & 36 & 46 & 34 & 59 \\
\hline $\begin{array}{l}\text { Muy } \\
\text { Bajo }\end{array}$ & 58 & 157 & 58 & 84 & 118 & 54 & 52 \\
\hline
\end{tabular}

Fuente: elaboración propia.

Los resultados relacionados con el nivel de estrés en el personal administrativo de la Corporación Universidad de la Costa, presentados en la tabla 2, demuestran que 31 de los docentes encuestados presentan un nivel de estrés muy alto 37 , uno alto; 34 , uno medio; 42 , uno bajo y 83 , uno muy bajo. 


\section{Revista Electrónica Enfermeria Actual en costa Rica}

Tabla 2. Corporación Universidad de la Costa. Distribución del nivel de Estrés en el personal administrativo.

\begin{tabular}{lccccc}
\hline & Muy Alto & Alto & Medio & Bajo & Muy Bajo \\
\hline $\begin{array}{l}\text { Personal } \\
\text { administrativo } \\
\text { de la CUC }\end{array}$ & 31 & 37 & 34 & 42 & 83 \\
\hline
\end{tabular}

\section{DISCUSIÓN}

Tras observar que la mayor población pertenece al género femenino (un 69\%) es importante mencionar las consideraciones de Sánchez (2010) cuando expresa que las mujeres trabajadoras asumen doble jornada laboral, entendida como el salir de su hogar para unirse a la fuerza laboral y mejorar sus condiciones de vida y las de su familia, lo cual implica que a pesar de su ánimo por abrirse a nuevas oportunidades de trabajo y estudio, no pueden desligarse de sus labores domésticas, en tanto es muy probable que generen niveles elevados de fatiga laboral y estrés.

La cantidad de administrativos solteros es mayor que los casados, viudos, separados y en unión libre. Al respecto, Villegas y Santamaría (1999) comentan que los sujetos casados suelen tener un estilo de vida más estable y una perspectiva de vida mejor que la de los solteros, considerando, además, que los sujetos solteros podrían tender a usar el trabajo como fuente de vida social y esto le llevaría a implicarse excesivamente con la gente del ámbito laboral, por tanto, a un mayor riesgo de sufrir de síndrome de quemarse en el trabajo, entre otros aspectos que sobreponen niveles de estrés en este tipo de trabajadores. A lo anterior se unen los mayores porcentajes de personas con un estrato social que van desde el nivel 3 hasta el nivel 1, lo cual devela que su condición económica es más comprometida y de necesidades de supervivencia mayor a los demás, lo que a su vez contribuye en la manifestación del estrés en general.

Londoño et al. (2010) explican que los problemas de vivienda son un factor de riesgo para la depresión, dado que la falta de hogar, de vivienda adecuada, el vecindario insalubre, los conflictos de vecinos o propietarios, son condiciones básicas y al no tenerlas resueltas en la edad adulta se vive como una pérdida de ideales y puede afectar el estado de ánimo. En el contexto colombiano, estos problemas también se refieren a la dificultad para llevar al día los créditos hipotecarios, y los gastos ligados a la vivienda, como el alto porcentaje del salario que ocupan los servicios públicos. La depresión se produce por pérdida de personas, objetos e ideales, ya que poseer vivienda es un ideal social en esta cultura.

En cuanto al Desplazamiento vivienda-trabajo-vivienda, el porcentaje de factor de riesgo asociado en la población de estudio, evidencia niveles que sobrepasan al 75\% cuando se suman los niveles bajo, medio, alto y muy alto. Esta situación, de acuerdo con lo planteado por Gutiérrez y García (2005), incrementa el nivel de estrés en los trabajadores. Igualmente, el factor económico se evidencia en niveles de factor de riesgo que elevan el estrés de los trabajadores, cuando las cifras en porcentajes sobrepasan más del 75\%, lo cual agrupa los niveles que requieren intervención, como lo son bajo, medio, alto y muy alto., respecto de lo cual expresan Gómez y

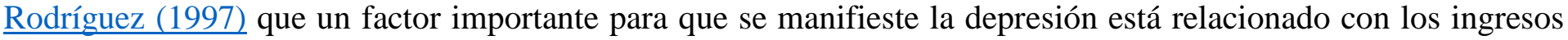
familiares. 


\section{Revista Electrónica Enfermeria Actual en costa Rica}

\section{CONCLUSIONES}

Ha sido posible caracterizar a los factores de riesgo psicosocial extralaborales, presentes en el personal administrativo de la Corporación Universidad de la Costa - CUC. En primer lugar, prevaleció el factor de riesgo "desplazamiento vivienda-trabajo-vivienda"; en segundo lugar, se presentó la "situación económica del grupo familiar", en tercer lugar, las "características de la vivienda y su entorno". Otro factor de riesgo con cifras relevantes para un cuarto lugar, fue el de "tiempo fuera del trabajo" y en quinto, la "influencia del entorno extralaboral sobre el trabajo". Finalmente, en los niveles más bajos, se ubica los factores de riesgo de "relaciones familiares" y de "comunicación y relaciones interpersonales".

El personal administrativo que labora en la institución está expuesto a los factores de riesgo psicosocial extralaboral y niveles de estrés significativos, que desprenden un llamado de atención, frente a las orientaciones ministeriales sobre los problemas epidemiológicos derivados de tales resultados, a partir de los cuales la institución puede encaminar su gestión con la intervención oportuna, para solventar las situaciones emergentes y recrear un clima laboral y de convivencia armónica para todos.

\section{Conflicto de Intereses.}

Las autoras declaran que no existe conflicto de intereses en el desarrollo de este estudio.

\section{REFERENCIAS BIBLIOGRÁFICAS}

Aguirre, E. y Guevara, G. (2012). Factores asociados con el desempeño del personal que labora como oficiales de tránsito y seguridad en la universidad de costa rica. Revista Enfermería Actual de Costa Rica, 22, 1-10.

Arias, F., Barboza, N., Blanco, J., Fajardo, E., Rivera, R., Rodríguez, S., Ugalde, S. y Álvarez, R. (2008). Síndrome del desgaste profesional o de burnout en el personal de enfermería. Revista Enfermería Actual de Costa Rica, 13, 1-10.

Cámara Colombiana de la Infraestructura (2005). Movilidad ¿una crisis sin solución? . Recuperado de http://www.infraestructura.org.co/historial/noticia9_052807.php

Cano, V. (2012). Identificación, medición y evaluación de los factores de riesgo psicosocial, en el personal de trabajadores de la Universidad San Francisco de Quito (tesis de posgrado). Universidad San Francisco, Quito, Ecuador.

Congreso de Colombia (1992). Ley 30 de 1992. Por la cual se organiza el servicio público de la educación superior. Diario Oficial 40700, Bogotá, Colombia.

Consejo Nacional de Acreditación - CNA (2006). Lineamientos para la acreditación institucional. Recuperado de: http://cms-static.colombiaaprende.edu.co/cache/binaries/articles-186359 lineamientos 3.pdf?binary rand=2342

Castillo, M. y Villalobos, F. (1996). Cuestionario para la evaluación del estrés. Bogotá: Ministerio de Trabajo y Seguridad Social.

Díaz, D. (2011). Estrés laboral y sus factores de riesgo psicosocial. Rev. CES Salud Pública, 2(1), 80-84. 


\section{Revista Electrónica Enfermeria Actual en costa Rica}

Fernández, B. (2012). Factores de Riesgo Psicosocial - Seguridad en el trabajo, Higiene industrial, Ergonomía y Psicosociología aplicada (tesis de posgrados) Universidad Internacional de la Rioja, Madrid, España.

Gil-Monte, P. (2009). Algunas razones para considerar los riesgos psicosociales en el trabajo y sus consecuencias en la salud pública. Rev. Esp. Salud Publica, 83(2), 169-173.

Gil-Monte, P. (2012). Riesgos Psicosociales en el trabajo y en la salud ocupacional. Rev. Salud pública, 29 (2), 237-241.

Gómez, C. y Rodríguez, N. (1997). Factores de riesgo asociados al síndrome depresivo en la población colombiana. Rev. Col. Psiquiatría, 26(1), 23-35.

Guerrero, J. (2003). Los roles no laborales y el estrés en el trabajo. Revista Colombiana de Psicología, 12, 73-84.

Gutiérrez, J. y García, J. (2005). Cambios en la movilidad en el área metropolitana de Madrid: El creciente uso del transporte privado. Anales de geografía de la universidad Complutense, 25, 331-351.

Hernández, P., Soria, M. y Silla, J. (2003). El Estrés Laboral: ¿Un Concepto Cajón-De-Sastre? Revista Relaciones Laborales, 10(11), 167-185.

Instituto Nacional de Seguridad e Higiene en el Trabajo (2001). V Encuesta Nacional de Condiciones de Trabajo. Ministerio de trabajo y Asuntos Sociales (INSHT). Madrid.

Lazarus, R. (1999). Stress and Emotion: A New Synthesis. New York: Springer Publishing Company.

Londoño, N.H., Juárez, F., Palacio, J., Muñiz, O., Agudelo, D., Marín, C.A., Lemos, M., Toro, B.E., Ochoa, N.L., Hurtado, M.H., Escobar, B., Herrón, I., Gómez, I., Uribe, A.F., Rojas, A.L., Pinilla,M.L., Villa-Roel, D., Villegas, M.J., Arango, A.L., Restrepo, P.A. y López, I.C. (2010). Factores de riesgos psicosociales y ambientales asociados a trastornos mentales. Suma psicológica, 17(1), 59-68.

Mamani, A., Obando, R., Uribe, A. y Vivanco, M. (2007). Factores que desencadenan el estrés y sus consecuencias en el desempeño laboral en emergencia. Rev. Per Obst. Enf, 3(1), 50-57.

Marulanda, I. (2007). Estrés laboral enemigo silencioso de la salud mental y la satisfacción con la vida. Recuperado de: http://www.slideshare.net/vladimirvargass/marulanda-i-2007-estres-laboral-enemigo-silencioso-de-la-salud-menta

Ministerio de Educación Nacional (1992).Ley 30, Por la cual se organiza el servicio público de la educación superior. Recuperado de: http://www.alcaldiabogota.gov.co/sisjur/normas/Norma1.jsp?i=253

Ministerio de la Protección Social, MPS. (2010). Batería de Instrumentos para la Evaluación de Factores de Riesgo Psicosocial, Resolución 2646 de 2008. Recuperado de:

http://fondoriesgoslaborales.gov.co/documents/Publicaciones/Estudios/Bateria-riesgo-psicosocial-1.pdf

Ministro de la Protección Social (2008). Resolución 2646 de 2008, por la cual se establecen disposiciones y se definen responsabilidades para la identificación, evaluación, prevención, intervención y monitoreo permanente de la exposición a factores de riesgo psicosocial en el trabajo y para la determinación del origen de las patologías causadas por el estrés ocupacional. Diario Oficial 47059, Bogotá, Colombia. 


\section{Revista Electrónica Enfermeria Actual en costa Rica}

Moreno-Jiménez, B. y Garrosa-Hernández, E. (2009). Globalización y riesgos laborales emergentes. Ciencia \& Trabajo, 11(32), 31-35.

Organización Internacional del Trabajo.(1998). Declaración Sociolaboral de Mercosur. Recuperado de http://www.ccscs.org/index.php/documentos-ccscs/participacion-en-el-mercosur/item/534-declaraci\%C3\%B3n-sociolaboral-del-mercosur.

Organización Internacional del Trabajo - OIT (2006). C187 - Convenio sobre el marco promocional para la seguridad y salud en el trabajo. Recuperado de http://www.ilo.org/dyn/normlex/es/f?p=NORMLEXPUB:12100:0::NO::P12100_INSTRUMENT_ID:312332

Orozco, O. (2010). Factores psicosociales del ambiente laboral que caracterizan a funcionarios con contratación a término fijo e indefinido de una universidad privada de la ciudad de Cali. Revista Científica Guillermo de Ockham, 8(2), 5568.

Presidencia de la República (1984). Decreto 614 de 1984, por el cual se determinan las bases para la organización y administración de Salud Ocupacional en el país. Diario Oficial 36561.

Sánchez, O. (2009). Factores intra y extra laborales de los y las trabajadoras de una empresa pública de Costa Rica. Revista Enfermería Actual de Costa Rica, 17, 1-9.

Sánchez, O. (2010). Factores intra y extra laborales de los y las trabajadoras de una empresa pública de Costa Rica. Rev. Enfermería Actual en Costa Rica, 17, 1-9.

Sotillo, R. (2000). Estrés en Emergencias Sanitaria, Recuperado de http://www.google.com/url?sa=t\&rct=j\&q=\&esrc=s\&source=web\&cd=2\&ved=0CDIQFjAB\&url=http $\% 3 \mathrm{~A} \% 2 \mathrm{~F} \% 2$ Fwww.capitalemocional.com\%2FTextos_Psico\%2FESTRES1.doc\&ei=PYCGU6OwBo2wsATGkoC4BA\&usg=AF QjCNFb9rzyXo07YhImoOYuZGC-PXwhdw\&bvm=bv.67720277,d.cWc

Villegas, J. y Santamaría, E. (1999). Evaluación del nivel de Burnout en una muestra de trabajadores del área de tratamiento de un Centro Penitenciario. Revista Española de Sanidad Penitenciaria, 1(3), 68-72. 